\title{
L'évolution des approches du décrochage scolaire
}

\author{
Françoise Bruno \\ Aix-Marseille Université, ADEF \\ Francoise.Bruno@ac-nice.fr \\ Frédéric Saujat \\ Aix-Marseille Université, ADEF \\ frederic.saujat@univ-amu.fr \\ Christine Félix \\ Aix-Marseille Université, ADEF \\ marie-christine.felix@univ-amu.fr
}

Dans un contexte de massification de l'enseignement et de difficultés d'insertion sur les marchés du travail, le décrochage scolaire est devenu depuis vingt ans un problème majeur de tous les systèmes scolaires dans les pays industrialisés (Blaya, 2013). Particulièrement pour les populations possédant un faible niveau de qualification, la lutte et la prévention contre le décrochage ont été progressivement présentées comme des enjeux prioritaires de politique publique en termes d'éducation. Ainsi, les chiffres concernant les early school leavers (les élèves qui quittent l'école précocement) constituent aujourd'hui un indicateur pour le pilotage des actions entreprises au niveau des politiques publiques mais aussi des établissements scolaires.

Les travaux de recherche concernant le décrochage sont nombreux, et la plupart d'entre eux visent à qualifier et à quantifier le phénomène, ainsi qu'à en repérer les causes, afin d'envisager des solutions pour en réduire l'impact (Lehr et al., 2003). Ces travaux convergent sur le fait que le décrochage scolaire est multidimensionnel, que ses origines sont multiples et peuvent être éclairées par des cadres théoriques de différentes disciplines (Robertson, Collerette, 2005). Par exemple, les études en psychologie mettent surtout l'accent sur les dimensions intrapsychiques, comportementales et socio-interactionnelles, alors que les chercheurs en éducation se penchent davantage sur les liens entre le décrochage, les méthodes pédagogiques et l'environnement éducatif (Janosz, 2000). Des sociologues et des historiens s'intéressent également à l'abandon scolaire, dont ils examinent la problématique sous l'angle de la construction sociale du phénomène, des facteurs structurants des institutions ou encore du rapport entre les classes sociales (Dorn, 1996). Les représentants d'un courant davantage «culturaliste » considèrent de leur côté que la non-maîtrise des habiletés sociales et personnelles exigées par l'école (mais pas systématiquement enseignées explicitement), est déterminante dans le processus de décrochage (Bautier, Rochex, 1997 ; Bonnéry, 2003). Ces approches sont associées plus récemment à des tentatives de compréhension davantage systémiques et dynamiques, combinant des facteurs socio-culturels et socio-économiques, scolaires et personnels (Blaya, Fortin, 2011).

Deux problèmes principaux émergent de ce phénomène en évolution et de sa prise en compte. Premièrement, il existe des obstacles à son évaluation (Rumberger, 2001). En effet, même si le taux d'abandon apparaît comme un indicateur objectif et relativement facile à mesurer, le 1 
processus est finalement complexe, chaque parcours semble particulier et requiert des études longitudinales (Blaya, 2009). De même, il est difficile de repérer, parmi la multitude de facteurs de risque de décrochage, ceux qui opèrent de manière significative, directement ou indirectement, tant ces facteurs sont interdépendants et liés entre eux (Balas, 2012). Deuxièmement, après une nette diminution de l'abandon scolaire correspondant aux premières politiques structurelles de massification de l'enseignement, on constate peu d'évolution des taux, notamment dans les pays industrialisés (Commission Européenne, 2011). Ce constat se double d'une impression d'inefficacité globale des programmes de prévention et de lutte contre le décrochage, qui parviennent à contenir partiellement le phénomène mais sans aboutir la plupart du temps à une stratégie efficace de prévention primaire de l'abandon (Colombo, 2010). Comment expliquer cette tendance ? Est-elle en rapport avec la difficulté d'évaluation évoquée plus haut qui rendrait difficile l'identification des leviers d'action et empêcherait de possibles régulations ? Est-elle simplement la conséquence d'une définition imprécise du phénomène (autrement dit : parle-t-on toujours de la même chose quand on parle de décrochage scolaire) ? Les études réalisées dans ce domaine, majoritairement descriptives et probabilistes (Lehr et al., 2003), suffisent-elles pour servir de base à une compréhension fine de ce qui pousse les jeunes à décrocher des cursus scolaires?

En France, à l'heure où l'institution demande avec insistance au système scolaire de prendre en charge tous les élèves et de prévenir le décrochage, ces questions semblent constituer une source de malaise au sein du milieu enseignant (Bruno, Méard, Walter, 2013). Dans le cadre d'une recherche-intervention à visée transformative portant sur le développement du pouvoir d'agir (Clot, 2008) d'enseignants de collège confrontés au risque de décrochage scolaire chez les élèves, il nous a paru nécessaire de réaliser un tour d'horizon des travaux relatifs à ce sujet dans la littérature internationale (principalement européenne et nord-américaine) durant les 20 dernières années. Le but est donc ici de mieux cerner les éléments de contexte susceptibles d'infléchir la situation de travail de ces professionnels (ce que Clot (2007) définit comme « la dimension transpersonnelle du métier »). Il s'agit pour nous, au-delà d'une prise en compte de ces travaux de référence à dimension majoritairement sociologique et psychologique, de nous positionner dans une recherche fondamentale de terrain (Clot, 2008) permettant aux participants de reprendre la main sur une situation de travail qui, souvent, semble leur échapper.

\section{Méthode}

Les mots-clés «school dropout», "early school leavers", "at-risk students », "school leaving », « décrochage scolaire », «abandon scolaire», «désaffiliation scolaire », «déscolarisation», «absentéisme», ont été utilisés pour sélectionner les publications présentées dans cette revue de littérature. Ces termes ont permis de distinguer les textes traitant directement du décrochage scolaire, ainsi que d'autres ayant pour objet d'autres problématiques associées au phénomène de décrochage, en amont ou en aval (comme par exemple la délinquance ou la dépression chez l'adolescent). Les moteurs de recherche suivants ont été utilisés : Science Direct, Educational Resource Information Center, Taylor \& 
Francis, Google Scholar, pour les articles anglophones, Cairn et Revues.org pour les articles francophones. La recherche par mots-clés a été également étendue aux sites des revues classées par le HCERES (Haut conseil de l'évaluation de la recherche et de l'enseignement) en sciences de l'éducation. Nous avons choisi de ne pas présenter ici les articles traitant des programmes de prévention et de lutte contre le décrochage scolaire qui, du fait de leur nombre, auraient nécessité une publication à eux seuls. Nous avons également écarté de cette revue les textes centrés sur les problématiques d'échec scolaire qui ne faisaient pas directement référence au lien entre difficultés scolaires et décrochage, toujours dans un souci de concision. Enfin, nous avons inclus dans cette revue les ouvrages scientifiques traitant directement de la question du décrochage scolaire.

L'empan temporel choisi (les 20 dernières années) correspond en France à l'inflexion des politiques éducatives repérée par Broccolichi (1995) dans le sens d'une massification de la scolarisation et d'un allongement des durées d'études, en même temps qu'un objectif de réussite de tous les élèves dans le cadre d'un collège unique. Le «nouveau contrat pour l'école» (loi 95-836 du 13 juillet 1995) instaure, outre un pilotage par les objectifs, une obligation de doter chaque jeune d'une formation qualifiante à leur sortie du système scolaire par le biais d'une prise en charge massive de tous et de réponses adaptées aux élèves en difficulté. Dans un contexte de crise économique et sociale fragilisant les jeunes les moins qualifiés sur le marché de l'emploi, la déscolarisation est perçue à partir de cette période comme une forme avancée de l'échec scolaire (Bonnéry, 2001 ; 2004a). Par ailleurs depuis les années 1995, ce phénomène n'est plus considéré comme un problème marginal touchant une frange de la population scolaire, mais au contraire comme un risque pouvant potentiellement toucher chaque élève à un moment de son parcours (Bonnéry, 2004a).

Cette démarche nous a conduits à identifier et analyser 85 articles et 21 ouvrages et rapports relatifs aux contextes français (33) et international, principalement européen (15), nordaméricain (59), australien et néo-zélandais (3). La prédominance de ces zones (Europe et Amérique du Nord), que nous avions attribuée initialement aux référencements préférentiels des moteurs de recherche d'articles, trouve son origine, comme nous le verrons dans les résultats, dans le fait que la question du décrochage préoccupe quasi exclusivement les pays industriels confrontés au paradoxe d'une élévation du niveau d'instruction et de difficultés accrues d'insertion dans le marché du travail. Ces pays sont principalement européens et américains du nord (ainsi que les pays économiquement développés en Océanie, dans une moindre mesure).

\section{Résultats}

Nous observerons tout d'abord comment émerge le terme de « décrochage scolaire » dans la littérature scientifique ; après avoir examiné comment les auteurs définissent ce terme et avoir montré que celui-ci recouvre en réalité une grande diversité de situations, nous dégagerons les principales conceptions de ce phénomène, grâce à l'analyse des travaux relatifs aux typologies d'élèves, et leur évolution, d'une vision statique à tendance épidémiologique vers une compréhension plus dynamique, basée sur des études longitudinales. Puis nous discuterons 
des intérêts et des limites des approches par facteurs de risque et facteurs de protection. Nous envisagerons enfin une orientation de recherche complémentaire pour comprendre l'intimité des processus en classe et restaurer le pouvoir d'agir des principaux acteurs de la prévention du décrochage scolaire.

\section{Un terme fondamentalement ambigu}

De quoi parle-ton lorsqu'on évoque le décrochage scolaire? L'ambiguïté de ce terme, nonobstant les définitions institutionnelles, participe de ce que l'expression commune appelle le «malaise enseignant ». Nous tenterons de montrer que l'émergence de la problématique du décrochage est corrélée au contexte socio-économique et à sa prise en compte dans les politiques éducatives des pays développés, en même temps qu'à l'assignation au système scolaire de nouvelles missions : assurer la cohésion sociale et préparer l'entrée des jeunes dans les marchés du travail. Le décrochage devient par conséquent un indicateur de mesure de l'efficacité de ces politiques publiques d'éducation et d'enseignement, sans que les implicites et les contradictions du vocable aient tous été levés. Cette confusion ajoute par là-même une difficulté de prise en compte de la prescription : il est essentiel de combattre le décrochage, mais comment en cerner concrètement les contours ?

\section{Emergence du décrochage scolaire et contexte socio-économique}

Le décrochage scolaire n'est pas un phénomène nouveau, mais demeurait peu visible tant que le marché du travail absorbait une main d'œuvre peu qualifiée. Ainsi, en France, jusqu'à la fin des années 1970, le marché du travail absorbait environ 200000 jeunes non qualifiés, sortis prématurément du système scolaire (Glasman, 2011). Dans les années 1960 et 1970, les pays anglo-saxons furent les premiers à présenter une vision structurelle du décrochage scolaire, définie par les «taux d'inachèvement » (underachieving), calculant le rapport entre les élèves de bas rendement scolaire et les taux moyens de rendement, ainsi que les abandons précoces. Le différentiel était alors expliqué par la privation socio-culturelle et la marginalité socioéconomique (rapport Coleman et al., 1966). À la fin des années 1970, une vision élargie du décrochage scolaire aboutit à une conception émergente, dans un contexte scolaire et extrascolaire de malaise de la jeunesse, de carence de régulation et de socialisation. On constate à cette époque un début de prise en compte de ceux qui se maintiennent dans le système de manière irrégulière, et qui présentent des carences de motivations et de compétences (Ribolzi, 1984). Des modèles systémiques, basés sur les facteurs socio-culturels, socio-économiques, scolaires, personnels sont alors conçus dans le but de garantir à l'école une meilleure capacité de rétention. Parallèlement, la tendance de fond des politiques publiques en matière éducative dans les pays industrialisés a été de donner la priorité à l'ouverture de l'école au plus grand nombre, favorisant l'accueil de nouveaux publics (par exemple le collège unique pour le contexte français), par la création de nouvelles filières et de dispositifs de lutte contre l'échec scolaire. De plus, au-delà de la question initiale de l'absentéisme (Blaya, 2009), depuis le milieu des années 1980, l'école a donc dû prendre en compte la question de l'insertion professionnelle et de son accompagnement (Bernard, 2011a; Blaya, 2013). Cet objectif a alors pris une part de plus en plus importante dans les pratiques scolaires, et est devenu un enjeu majeur de transformation du système d'enseignement. En France par exemple, le 
collège unique a dû s'adapter aux nouveaux publics et accompagner les jeunes en difficulté, en particulier par les formations en alternance et les dispositifs d'insertion. Depuis 1984, l'éducation nationale est appelée à conserver et à prendre en charge les jeunes voués à une sortie précoce, ce qui a entraîné une redéfinition des modes d'intervention dans et hors de l'école. Dès 1986, le Dispositif d'Insertion des Jeunes de l'Éducation Nationale (DIJEN), remplacé ultérieurement par la Mission de Lutte contre le Décrochage Scolaire (MLDS) fut créé pour aider à la prise en charge des jeunes sans diplôme (recherche d'emplois, préparation de diplômes). Dans une optique expérimentale, le soutien des courants pédagogiques engagés dans la lutte contre l'échec scolaire fut sollicité (Geay, 2003).

Depuis les années 1990, le terme «décrochage scolaire » a peu à peu remplacé celui d' « échec scolaire» dans les discours institutionnels (Geay, 2003). Ce glissement sémantique semble révéler une volonté de la part des instances de pilotage d'articuler le « dedans » et le « dehors » de l'école (Glasman, Oeuvrard, 2011). Par ailleurs, face à des problématiques de plus en plus pressantes de restauration de la cohésion sociale et de contention de la violence (Douat, 2011 ; Thin, 2002), le décrochage est apparu comme la part visible de préoccupations débordant le cadre strictement scolaire (Millet, Thin, 2005). Ce phénomène est devenu depuis cette époque d'autant plus perceptible dans les pays industrialisés en Europe occidentale, en Amérique du Nord et en Australie, que la tension continuait de s'accentuer entre l'élévation du niveau d'instruction et les difficultés d'insertion dans le marché du travail (Colombo, 2010 ; Hayden, Blaya, 2008 ; Marks, Fleming, 1999 ; Rumberger, Lamb, 2003 ; Vanttaja, Jarvinen, 2006). Selon nous, cet élément contextuel permet de comprendre la multiplication des financements de travaux de recherche et donc des publications dans ces zones géographiques.

Paradoxalement, la population concernée par le décrochage scolaire est aujourd'hui nettement moins nombreuse que dans les années 1970 ; en France par exemple, les sortants non qualifiés représentaient 35\% de la population scolaire en 1965, contre 6\% en 2005 (Bernard, 2007 ; 2011b). En revanche, les conséquences du décrochage apparaissent de plus en plus problématiques dans la mesure où l'éducation est progressivement érigée comme un élément central du bien-être individuel et collectif, et le diplôme comme un passeport pour l'emploi (Marcotte, Lachance, Lévesque, 2012). Par ailleurs, le coût du décrochage est régulièrement avancé pour justifier la mise en œuvre des politiques publiques, qu'il soit financier (dépenses des collectivités publiques, manque à gagner en taxes et revenus non perçus, programmes en direction des personnes sans emploi, prévention et traitement de la criminalité) (Christenson, Thurlow, 2004; Malo, Sarmiento, 2010) ou social (difficultés à accéder à un emploi, nécessité de recourir aux aides sociales, comportements déviants, délinquance) (Battin-Pearson et al., 2000 ; Fortin, Picard, 1999 ; Jimerson, Anderson, Whipple, 2002). Le décrochage scolaire est donc identifié comme « une maladie nosocomiale de l'école » (Tièche-Christinat, 2015) qu'il convient de combattre.

\section{Un même terme pour désigner des situations différentes}

Si le concept de décrochage s'imposant peu à peu pouvait laisser penser à une époque qu'il était possible d'embrasser l'ensemble des problématiques éducatives et en même temps les 
réduire à des chiffres et des courbes, on se rend compte en fait que sa dimension englobante ne parvient à gommer ni la complexité ni la variété des phénomènes. Certes, le remplacement progressif de la notion d'échec par celle de décrochage scolaire amène à dissiper la vision d'un abandon limité aux seuls élèves ayant de mauvais résultats scolaires et prioritairement issus de milieux défavorisés (Franklin, Streeter, 1995). Mais ce glissement notionnel impose peu à peu une conception multiforme de la population concernée, présentant des caractéristiques disparates et invoquant des raisons variées pour expliquer leur décision de quitter l'école. Le terme de décrochage scolaire peut par exemple recouvrir aussi bien les situations d'exclusion du jeune par le système scolaire, que l'absentéisme prolongé, jusqu'au choix volontaire d'abandonner les études (Blaya, 2010). Les circonstances d'abandon scolaire sont également disparates, et les élèves concernés arborent des profils personnels, familiaux et scolaires diversifiés (Aloïse-Young, Chavez, 2002; Rumberger, Lim, 2008). En France, la notion de «décrochage de l'intérieur », développée notamment par Bonnéry (2003; 2004a), Broccholichi, Ben Ayed (1999), dans la continuité des travaux de Bourdieu et Champagne (1993), qualifie des élèves qui, sans présenter de situation d'absentéisme caractérisé, se trouvent dans un processus de désaffiliation progressive et souvent silencieuse, lié notamment à des malentendus nés des écarts entre la culture scolaire et l'environnement économique, social et familial, alors que les systèmes anglo-saxons ont plutôt mis l'accent sur les coûts sociaux du décrochage, comme la dépendance, la criminalité et la faible productivité économique (Dorn, 1996).

La dimension multiforme du terme de décrochage est accentué par le fait que les définitions règlementaires du décrochage peuvent varier selon les Etats, les districts (Rumberger, 2001), selon le niveau minimal de qualification pris en compte, enfin selon l'indicateur de mesure retenu : pourcentage d'élèves ayant quitté l'école par rapport au nombre d'inscrits dans un niveau scolaire, ou pourcentage d'élèves d'un âge donné n'ayant pas atteint un niveau minimum requis et n'étant plus inscrits en formation, ou encore pourcentage d'élèves ayant décroché dans une classe d'âge donnée (Natriello, 1997). Le calcul des taux de décrochage est également sujet à des variations selon la période de l'année où sont effectués les relevés, le degré d'instruction pris en considération, la méthode utilisée pour comptabiliser les absences et les abandons. Ces éléments jettent le doute sur le bien-fondé de l'utilisation de ces chiffres comme éléments de comparaison internationale (Colombo, 2010). Ainsi, la notion de décrochage est englobante et, pour cette raison, elle peut laisser penser que l'on tient enfin «l'outil » de mesure et de compréhension des problématiques éducatives et de l'articulation entre l'école et la société. Mais elle véhicule néanmoins la complexité de ces problématiques et, justement parce qu'elle est englobante, fait courir le risque de les niveler.

\section{Le décrochage scolaire, indicateur de performance des politiques éducatives}

Pour illustrer ce risque, force est de constater que le décrochage scolaire est actuellement perçu comme un des indicateurs principaux du niveau d'équité des systèmes éducatifs, et par conséquent un outil de mesure des politiques éducatives, malgré les limites que nous avons soulignées (Colombo, 2010). Les différents systèmes nationaux de l'Union Européenne sont ainsi supposés converger vers une diminution des taux de décrochage, grâce aux 
comparaisons qu'offrent les évaluations PISA, les données statistiques d'EUROSTAT (Bernard, 2011a ; Zay, 2005). En Amérique du nord également, le décrochage est considéré comme indicateur de réussite ou d'échec des politiques éducatives (Chapman et al., 2011).

Le taux de décrochage scolaire est également utilisé pour mesurer les performances des établissements scolaires (Lehr et al. 2003), même s'il faut souligner que ceux qui affichent les meilleurs résultats au regard des taux de diplomation ne sont pas nécessairement ceux qui réussissent à réduire le décrochage. En effet, certains établissements, devant des élèves à risque de décrochage présentant des résultats scolaires plus faibles que la moyenne, peuvent être amenés à conduire une politique dissuasive, par exemple, en les encourageant à se tourner vers des formations non diplômantes ou même à changer d'école; ce qui permet ainsi de ne pas les comptabiliser dans les statistiques concernant les résultats aux évaluations ou les taux de diplomation (Rumberger, Palardy, 2005). Là encore, on touche du doigt les limites et les possibles effets pervers de chiffres ou de courbes qui réduisent de la sorte la réalité de l'abandon et de la persévérance scolaires.

\section{Une prise en compte progressive de la complexité du phénomène}

Face à l'émergence du phénomène de décrochage scolaire et l'importance qui lui est accordée dans un contexte de massification de l'enseignement, il est apparu nécessaire de caractériser les publics concernés, dans le but d'orienter les politiques publiques. En réponse à la commande de rapports officiels ou d'appels d'offre, de nombreuses équipes de recherche se sont polarisées sur l'analyse du décrochage du point de vue des élèves et sur les facteurs de risque auxquels les jeunes sont exposés.

\section{Le décrochage scolaire comme résultat d'un cumul de facteurs de risque}

Une première catégorie de travaux consiste donc à dresser un tableau le plus précis possible des élèves à risque de décrochage. Partant du constat que la population scolaire concernée par le décrochage est hétérogène, ces travaux se penchent sur les caractéristiques de ces élèves afin de mieux en cerner les profils, se situant en cela dans une approche de type épidémiologique (identification des publics à risque, tentatives d'isoler les variables). Par exemple, Fortin et al., (2006) établissent une typologie distinguant différents décrocheurs : antisocial, non-intéressé par l'école, présentant des difficultés d'adaptation sociales et scolaires, dépressif. Ces caractéristiques individuelles sont également présentes dans les travaux de Rumberger (2004) qui adjoint à ce cadre de compréhension une perspective institutionnelle, prenant en compte l'environnement familial, scolaire, communautaire, ainsi que l'influence des pairs. Le but de ces travaux est de déterminer les facteurs pouvant conduire au décrochage, afin de porter une attention particulière aux publics considérés comme «à risque ». Pour Fortin et al., (2004), le décrochage est corrélé à un cumul de facteurs de risques personnels, familiaux et scolaires. Les auteurs soulignent dans cette étude que le décrochage ne constitue pas un phénomène soudain et brutal, mais qu'il est l'aboutissement d'un processus sur le long terme, pouvant parfois même trouver son origine dès le plus jeune âge et l'enseignement préscolaire (Hammond et al., 2007), ce qui invite à 
s'interroger sur les facteurs de risque qui peuvent, au cours de ce processus, influencer le décrochage (Battin-Pearson et al., 2000 ; Rumberger, 2004 ; Rumerger, Lim, 2008 ).

\section{Une multiplicité de facteurs de risque}

Rumberger $(1995$; 2001) et Rumberger, Lim (2008) distinguent deux axes principaux dans l'analyse des facteurs de risque : l'axe des caractéristiques personnelles des élèves, et celui des caractéristiques institutionnelles (comprenant les facteurs liés à l'école, aux familles ainsi qu'aux communautés d'appartenance des élèves).

Parmi les facteurs de risque personnels, le genre apparaît comme discriminant dans de nombreuses études, les garçons étant en général plus exposés au décrochage que les filles (Fortin, Picard, 1999 ; Rumberger, 2004). Selon Sinclair et Fraser (2002), il apparaît que les filles ont en général une perception du climat scolaire plus positive que les garçons ; elles semblent tisser davantage de liens au sein de l'école, tant avec les pairs qu'avec les enseignants (Lessard et al., 2007). Les facteurs de risque principaux diffèrent donc pour les filles et pour les garçons : les premières sont exposées aux problèmes de comportement, au faible engagement scolaire, ainsi qu'aux médiocres résultats académiques, alors que les seconds subissent plutôt les effets des dysfonctionnements familiaux et des attitudes négatives envers les enseignants (Lessard et al., 2004).

Parmi ces recherches visant à déterminer les facteurs de risques du décrochage scolaire, un certain nombre met le focus sur la dépression de l'adolescent, facteur d'autant plus important que ces signes peuvent passer inaperçus auprès des adultes, parents et enseignants (Fortin et al., 2004 ; Hickman et al., 2008). Les symptômes dépressifs et leur corollaire, l'isolement social, présentent des effets négatifs sur les résultats scolaires, et conséquemment sur le risque de décrochage (Marcotte, Lévesque, Fortin, 2006), d'autant plus qu'ils sont souvent accompagnés d'une faible estime de soi et d'un sentiment d'incompétence (Bernard, 2007).

Les problèmes de comportement, souvent associés aux symptômes dépressifs (Fortin et al., 2006), sont également prédicteurs de décrochage. Les études de Cairns, Cairns et Neckerman, (1989), Hickman et al. (2008), Jimerson et al. (2002) pointent les difficultés comportementales précoces comme des signes annonciateurs de faibles résultats scolaires, et donc de décrochage. Le manque de motivation et un faible sentiment d'auto-détermination (Vallerand, Fortier, Guay, 1997) ainsi qu'un faible intérêt pour l'école et les études constituent également, selon Janosz et al. , (2008) et Hickman et al. (2008), un élément de fragilité prédisposant à l'abandon dès les premières difficultés. Le peu de valeur accordé par l'élève aux études et à la réussite scolaire accentue également le risque (Alexander, Entwisle, Kabbani, 2001).

Mais on pointe dès à présent les écueils auxquels sont confrontées ces études relatives aux facteurs de risque : les effets croisés et multiples de ces facteurs. En l'occurrence, ces signes dépressifs, associés à des problèmes comportementaux, sont présentés comme des éléments impactant la performance scolaire, sans pouvoir démêler l'effet en retour de ces performances scolaires sur l'état dépressif de l'adolescent. Ainsi les faibles résultats scolaires sont considérés comme élément principal du décrochage (Battin-Pearson et al., 2000 ; O'Connell, 
Sheikh, 2009) ; pour Fortin et al. (2013), celui-ci est le seul directement et significativement prédictif. Mais il est difficile de l'isoler de caractéristiques institutionnelles repérées par la communauté scientifique, notamment du contexte familial de l'élève, lui aussi identifié comme facteur de risque de décrochage par de nombreux auteurs (Battin-Pearson et al., 2000 ; Englund, Egeland, Collins, 2008 ; Jimerson et al., 2002): les relations entre les parents et leurs enfants apparaissent déterminantes dans le parcours des jeunes potentiellement décrocheurs. Lagana (2004) souligne le manque de cohésion entre les membres de la famille comme potentiellement fragilisant pour les adolescents; Fortin et al. (2006) pointent le manque de soutien émotionnel de la part des parents comme discriminant entre élèves décrocheurs et persévérants. La structure familiale est pointée de la sorte comme un facteur de risque pour Coudrin (2006), les enfants de familles monoparentales ou recomposées apparaissant comme plus exposés au décrochage, en lien de surcroît avec une fréquente mobilité résidentielle (Aston, McLanahan, 1994). Le niveau d'études des parents (en particulier celui de la mère) entre également en jeu (Hauser, Solon, Pager, 2004). BattinPearson et al. (2000) soulignent enfin que de faibles attentes parentales concernant la scolarité des enfants sont prédictives de faibles résultats scolaires, donc de décrochage.

Par ailleurs, le risque de décrochage scolaire semble aussi en rapport avec certaines caractéristiques sociales, en premier lieu les difficultés socio-économiques (Rumberger, 2001 ; Englund, Egeland, Collins, 2008). L'appartenance à une communauté ethnique minoritaire n'apparaît pas en soi comme facteur de risque, mais celle-ci étant souvent associée à un niveau socio-économique faible, les jeunes issus de ces minorités sont davantage exposés que les autres (Rumberger 2004). L'affiliation déviante constitue également un facteur exposant les jeunes au décrochage (Battin-Pearson et al., 2000 ; Cairns et al., 1989). Enfin Fortin et al. (2013) soulignent la corrélation entre l'appartenance à un milieu socio-culturel défavorisé et des relations parents-enfants peu développées, montrant à cette occasion qu'isoler différentes catégories de facteurs de risques s'avère difficile.

Si les études descriptives ont le mérite de révéler un tableau assez précis des élèves présentant un risque de décrochage afin d'aider les professionnels à les identifier, elles ne peuvent déterminer la part des éléments externes à l'école, tels que ceux pointés ici, de ceux qui relèvent des dispositifs scolaires eux-mêmes. Ainsi, de nombreuses études soulignent clairement que c'est l'institution scolaire elle-même qui est productrice de décrochage (Baker et al., 2001). Cette entrée permet de prendre de la distance vis-à-vis des déterminismes individuels et sociaux et d'aborder la question sous l'angle des interactions entre l'institution scolaire et les jeunes qui lui sont confiés. Par exemple, le taux de redoublement est identifié comme un déterminant directement prédictif du décrochage scolaire (Jimerson et al., 2002 ; Rumberger, 1995 ; 2001 ; 2004). En France, parmi ces situations de maintien d'élèves dans un niveau de classe, le redoublement du cours préparatoire constitue en particulier un facteur de risque majeur (Douat, 2011).

Selon Lee et Burkam (2003), c'est l'environnement scolaire global qui semble jouer un rôle dans le risque de d'abandon, spécifiquement lorsque l'école n'offre pas aux élèves un cadre clair, structuré et cohérent (Janosz, Leblanc, 1996) ou encore lorsque le contexte scolaire est 
dégradé (Bernard, 2007 ; Potvin et al., 2004). De fait, Cossette et al., (2004) ainsi que Fortin et al. (2006) notent que les jeunes à risque de décrochage perçoivent la classe comme un milieu peu organisé. Leurs relations négatives avec les enseignants ont de fortes conséquences sur l'échec scolaire et l'absence de persévérance (Davis, Dupper, 2004 ; Englund et al., 2008 ; Fortin et al., 2006, Murray, 2009). Ils ressentent un anonymat dans l'établissement et se perçoivent souvent comme invisibles et isolés (Rodriguez, 2010), éprouvant en outre des difficultés à nouer des relations avec leurs pairs (Fortin et al., 2013).

Ces multiples études sur les facteurs de risque, comme celles relatives aux typologies d'élèves décrocheurs que nous avons rapportées précédemment, donnent aux politiques et aux praticiens des repères permettant d'identifier des populations à risque et par conséquent de cibler les interventions dans leurs directions. Mais loin d'aboutir à un tableau clair et limpide du phénomène, elles pointent un grand nombre de facteurs étroitement liés entre eux. Au regard de notre démarche visant à restaurer le pouvoir d'agir des acteurs de l'éducation, nous pourrions craindre que ces tentatives d'identification systématique de facteurs de risque, qui ne parviennent pas finalement à distinguer ce qui relève de l'école de ce qui n'en relève pas, conduisent, à leur corps défendant, à «médicaliser» le phénomène et alimenter le fatalisme et la démobilisation de certains enseignants.

Certaines études empiriques permettent d'envisager les parcours d'élèves dans la durée, dans leur contexte scolaire, familial, économique, social. Elles prennent ainsi davantage en compte la complexité du phénomène et pointent l'organisation des systèmes scolaires elle-même comme étant à l'origine du risque de décrochage chez les élèves, au-delà de la probabilité de décrocher liée à leurs caractéristiques individuelles. Par exemple, les préjugés négatifs des enseignants à leur encontre influencent les performances des élèves de milieux populaires (Esterle-Hedibel, 2003) et, par conséquent, ont un impact sur le risque d'abandon. Rodriguez (2010) souligne que les enseignants ont de plus faibles attentes envers les élèves issus de ces milieux. Le niveau de formation et de qualification des enseignants semble également corrélé avec le degré de persévérance des élèves (Darling-Hammond, 1999 ; Zay, 2005) ; de ce fait, plutôt que les compenser, l'école semble renforcer l'impact des difficultés socio-culturelles des élèves (Blaya, 2010). Le processus d'orientation scolaire, s'il est perçu comme subi par l'élève, peut également conduire au décrochage (Blaya, 2010). Enfin les travaux concernant le décrochage cognitif apportent un éclairage sur ce que Broccolichi et Ben Ayed (1999), ainsi que Bonnéry (2003) nomment le «décrochage de l'intérieur». Dans cette perspective, les travaux de Bautier et al., (2002), Bonnéry (2003), Lessard et al. (2007), mettent en exergue les écarts entre la culture scolaire et celle de certaines catégories d'élèves. Des malentendus en résultent, conduisant, notamment lors de l'entrée dans le secondaire, à un processus de désaffiliation progressive. Bonnéry (2004b) montre même que c'est le fonctionnement de l'institution scolaire qui est remis en question à travers la problématique du décrochage, tant celle-ci révèle la difficulté des professionnels à maintenir certains jeunes dans un cursus unique jusqu'au terme de la scolarité obligatoire.

\section{D'une approche par facteurs de risque à l'identification de facteurs de protection}


Les recherches longitudinales, basées sur des observations sur un empan temporel long et qui tendent à décrire le décrochage scolaire comme un processus, mettent en évidence que certains des jeunes pourtant exposés à de multiples facteurs de risque de décrochage scolaire ne décrochent pas. Ce constat prometteur conduit un certain nombre de chercheurs à réaliser des études portant sur les «facteurs de protection» qui permettent de tracer des orientations d'action pour lutter contre l'abandon. Plusieurs équipes de chercheurs se sont donc penchées sur les différences entre facteurs discriminants entre « décrocheurs » et «persévérants », au sein de populations aux caractéristiques similaires (Fortin, Picard, 1999). Dans cette optique, Lessard et al., (2009) mettent en exergue le fait que les élèves considérés comme «à risque » et n'ayant pas décroché présentent un certain nombre de caractéristiques communes : de nombreuses relations sociales, un sentiment d'efficacité et d'estime de soi, une capacité à décider de leur trajectoire scolaire et professionnelle. Cette étude confirme les travaux de Knesting et Waldron (2006), qui déterminent comme facteurs de persévérance chez les jeunes l'orientation vers un but, la croyance qu'ils vont tirer profit de l'obtention d'un diplôme, une volonté de jouer les règles du jeu scolaire, et une relation signifiante avec un ou plusieurs enseignants qui croient en leur potentiel, les conseillent et se préoccupent de leur devenir (Fallu, Janosz, 2003). Blanchard et al., (2004) ainsi que Hardre et Reeve (2003), mettent en évidence le lien entre la motivation autodéterminée, de bonnes aptitudes scolaires et un faible taux d'abandon. Anderman (2002) met aussi en évidence le lien entre le sentiment d'appartenance à une communauté scolaire et la persévérance. Saint-Pierre, Denault et Fortin (2012) étudient le lien entre la participation des élèves à des activités para-scolaires et la diminution du risque de décrochage. Cette caractéristique est reprise par Blaya et al. (2011) qui préconisent le développement des «approches communautaires » favorisant chez les jeunes le développement de ce sentiment d'appartenance. O'Connell et Sheikh (2009) identifient des capacités qualifiées de «non-cognitives », comme la faculté de planification et la perception positive par les pairs, chez les adolescents à risque mais non décrocheurs. Enfin l'étude d'Englund et al. (2008) pointe l'aspect discriminant des relations parents-enfants et de l'implication des familles dans la scolarité des jeunes dans le processus de décrochage chez les publics à risque.

\section{Discussion}

Il semble ressortir de ce qui précède que l'appréhension du décrochage scolaire s'accommode mal d'une vision uniquement statique du phénomène, d'une perception à un instant « $\mathrm{t} »$. Cet instantané du décrochage, s'il permet de répondre à la demande des décideurs d'établir des statistiques et de chiffrer les performances relatives d'un établissement ou d'une politique éducative, ne prend pas en compte la complexité du phénomène ni son caractère dynamique.

\section{Vers une compréhension dynamique et contextualisée du décrochage scolaire}

Les études ayant pour but d'identifier les causes du décrochage et d'isoler des facteurs de risque, si elles présentent le mérite de caractériser les formes multiples du phénomène, nous semblent devoir être complétées par des approches plus contextualisées. La totalité des auteurs de référence aboutissent finalement à cette conclusion : ainsi, Rumberger (2001 ; 2004) identifie le décrochage comme un processus, trouvant ses racines dès l'école primaire. Alexander, Entwisle, Kabbani (2001) réalisent une étude considérant ce processus dans une 11 
perspective de parcours de vie. De même, l'étude de Blaya et Hayden (2003) comme celle d'Hickman et al. (2008), soulignent la précocité de ce processus et se penchent sur ses prémices dès l'enseignement préscolaire, et l'importance des moments de transition et de ruptures dans les parcours des jeunes concernés. Animés par la même préoccupation, Lan et Lantier (2003) mettent en évidence une dégradation d'un certain nombre de caractéristiques au fil des années, dans les parcours d'élèves décrocheurs : résultats scolaires, relations avec les enseignants, motivation, perception de l'environnement scolaire, participation aux activités scolaires et para-scolaires. En France, les travaux de l'équipe ESCOL et du réseau RESEIDA notamment, basés sur du matériau empirique (observations participantes, entretiens) se penchent sur le processus de décrochage cognitif à l'œuvre dès l'école élémentaire, en particulier chez les élèves dont la culture familiale est éloignée de la culture scolaire, principalement sur le plan de la langue orale et écrite (Bautier et al., 2002). Selon ces chercheurs, la prise en compte de la difficulté scolaire de ces élèves repose largement sur des malentendus (Bautier, Rochex, 1997), les enseignants constatant et déplorant cet écart culturel, mais ne mettant pas en place, la plupart du temps, d'apprentissages explicites permettant aux élèves d'intégrer les codes scolaires. Dès lors, le « décrochage de l'intérieur », c'est-à-dire la phase où le décrochage ne se traduit pas par des signes perceptibles de l'extérieur, est engagé dès l'école primaire. Cette observation rend cruciale la transition de l'enseignement primaire vers le secondaire, où l'opacité des codes, les liens plus distendus avec les enseignants, les nouveaux enjeux cognitifs et le relèvement des exigences lors de l'évaluation peuvent provoquer une réaction d'abandon et de désaffiliation en quelques jours seulement (Bautier et al., 2002 ; Bonnéry, 2003, 2004 ; Broccolichi, Ben Ayed, 1999).

Les données sur la transition entre l'école primaire et l'enseignement secondaire dans le devenir des élèves fragiles sont confirmées par les études de Lan et Lanthier (2003) et de Langenkamp (2010). Dès les premiers jours du collège, l'engrenage des problèmes inédits peut pousser certains jeunes à se réfugier dans des comportements de compensation, comme les bavardages, la provocation envers les adultes et les pairs. A travers cette approche cognitive du décrochage, on entrevoit que la classification des facteurs de risques en catégories (personnels, environnementaux, scolaires) n'est plus heuristique, tant ces composantes sont intimement liées et interdépendantes dans l'histoire de chaque jeune « décrocheur ».

\section{Corrélations et effets cumulatifs}

À cette dimension diachronique du décrochage telle que nous venons de l'évoquer ci-dessus, s'ajoutent les problèmes liés à une nécessaire prise en compte de l'enchevêtrement de causes multiples, qui relèvent, selon Glasman (2011), de différents registres de la vie du jeune (social, scolaire, émotionnel), révélant un processus multifactoriel impliquant les champs social, familial, éducatif, territorial, psychologique dans lesquels celui-ci évolue (Balas, 2012). Les effets cumulatifs de ces facteurs émergent de nombreuses études, comme les ruptures familiales répétées : déménagements, séparations, accidents de la vie, associées à des situations sociales précaires (Aston, Mac Lanahan, 1991 ; Millet, 2004). Battin-Pearson et al. (2000), Jimerson et al. (2002), Blaya et Fortin (2011) constatent l'effet cumulé des difficultés 
personnelles, sociales et scolaires dans l'accroissement du risque de décrochage, rendant de ce fait nécessaires les interventions multiniveaux pour prévenir et lutter contre le phénomène.

Comme nous l'avons signalé précédemment, cette dimension multifactorielle conduit plusieurs auteurs à pointer les limites des théories prédictives pour expliquer le décrochage scolaire (Fortin et al., 2013), tant les interactions entre facteurs de risques sont nombreuses : par exemple, ces auteurs remarquent que la dépression de l'adolescent, identifiée comme un facteur personnel, est corrélée avec une perception négative du climat de classe, sans qu'il soit possible de démêler, de ces deux caractéristiques laquelle serait la cause de l'autre. De même, la perception de la classe comme un milieu désorganisé par les élèves à risque pourrait provenir d'un déficit de sens lié à des lacunes concernant la connaissance du fonctionnement scolaire, mais aussi de la conséquence des perturbations engendrées par un climat familial peu sécurisant.

Finalement, la plupart des travaux pointe l'inextricabilité des facteurs de décrochage : par exemple, les interactions négatives entre professeurs et élèves sont largement corrélées avec de faibles résultats scolaires (Englund et al., 2008 ; Lessard et al., 2004). Ces derniers, considérés comme les principaux facteurs de risque et les seuls directement prédictifs du décrochage, peuvent eux-mêmes être compris comme résultant de facteurs sociaux, culturels, en lien avec l'organisation même des enseignements. Rumberger (2004) constate que si le domaine des facteurs scolaires semble le plus éclairant pour la compréhension du décrochage, il s'avère difficile, voire impossible, de démêler les effets des facteurs scolaires et individuels. Anderson, Hamilton et Hattie (2004) soulignent dans la même perspective l'influence du climat de classe sur les comportements de motivation et la réussite scolaire, montrant ainsi l'imbrication de ces facteurs de risques personnels et institutionnels.

Les facteurs de risque ne constituent donc pas isolément des prédicteurs du décrochage, cette approche pouvant conduire à mettre en œuvre des politiques de prévention ne ciblant pas les publics les plus concernés (Gleason, Dynarski, 2002). Colombo (2010) exprime ainsi des réticences sur la mise en œuvre de programmes établis à partir des facteurs de risque identifiés et déliés, cette approche conduisant davantage à des «politiques-pansements », plutôt qu'à une réelle prévention du décrochage par des politiques volontaristes et structurelles.

\section{Une nécessaire prise en compte de la complexité du phénomène}

Ces obstacles pour les chercheurs à dénouer la multiplicité des facteurs incitent plusieurs équipes de recherche à opérer un choix épistémologique consistant à contextualiser systématiquement le processus de décrochage, au lieu de l'essentialiser (Glasman, Oeuvrard, 2004 ; 2011). En effet, dans la mesure où il apparaît difficile, voire impossible, d'isoler les facteurs de risques, les approches corrélatives et cumulatives, voire qualitatives et compréhensives, paraissent plus heuristiques pour analyser la complexité du processus de décrochage scolaire. Ainsi, Esterle-Hedibel (2003) pointe dans une étude qualitative auprès de jeunes ayant décroché l'impact de l'institution scolaire et les effets d'étiquetage négatif, les liens entre les effets de bande et le processus de désaffiliation, les décalages entre les cultures familiale et scolaire. Dans la même veine, Millet et Thin (2007) font apparaître comment les 
différents parcours de ruptures scolaires des collégiens relèvent de processus à la fois singuliers et communs. En prenant appui sur des études de cas, Brown et Rodriguez (2009) reviennent sur le modèle de Rumberger (2004) qui dissocie facteurs individuels et institutionnels dans la compréhension du décrochage. Selon ces auteurs, les facteurs sont au contraire co-construits et liés entre eux. Par exemple, les effets sur le risque de décrochage du fait d'être d'origine défavorisée et latino-américaine (facteurs individuels) sont, selon ces auteurs, indissociables de la manière dont ces élèves sont perçus et traités à l'école (facteur institutionnel). Pour Rodriguez (2010), les facteurs de risque statiques deviennent variables en fonction des relations entre individus, structures et cultures, et perdent donc leur caractéristique déterministe (par exemple, le manque de motivation n'est plus considéré par cet auteur comme une caractéristique individuelle, mais comme dépendant de l'environnement dans lequel évolue l'élève : attentes de l'adulte, relations entre adultes et élèves, manière dont sont traités les centres d'intérêt des élèves à l'école).

Selon nous, c'est précisément dans la prise en compte d'une part de cet enchevêtrement de facteurs individuels et structurels, d'autre part de l'environnement dans lequel évoluent les élèves que réside une des pistes à venir les plus prometteuses pour comprendre le phénomène du décrochage scolaire. Dans cette perspective, les relations entre les perceptions et les actions individuelles d'une part et les facteurs scolaires d'autre part, méritent d'être interrogés conjointement et non de manière dissociée. Nous pouvons d'ailleurs constater que l'évolution des modèles explicatifs du décrochage par les chercheurs qui étudient depuis de nombreuses années le phénomène, comme Rumberger (2011) ou Fortin et al.(2013), va dans ce sens. Guigue (2013) préconise une approche «écologique»du contexte de classe et de vie des jeunes à risque de décrochage, un traitement uniforme ne pouvant s'appliquer à une population aussi diversifiée.

Il nous semble qu'aujourd'hui les chercheurs s'orientent principalement vers des approches ciblant non plus tant les caractéristiques des individus que l'organisation des systèmes scolaires et des politiques publiques, pour tenter de proposer des solutions de réduction du décrochage. Il en résulte des préconisations de mesures structurelles dans le but de favoriser la persévérance et l'accrochage scolaire pour l'ensemble des publics accueillis.

\section{Persévérance et accrochage scolaire : un regard sur la prévention de décrochage du côté des institutions}

À partir des années 2000, nous trouvons dans la littérature un certain nombre de travaux qui se penchent sur les notions de persévérance et d'accrochage scolaire, comme ceux de Blaya et al. (2011), Marcotte et al. (2011). Ces travaux sont initiés par le constat que c'est de la structure de l'institution scolaire elle-même que les évolutions des politiques de prévention du décrochage sont les plus prometteuses. En d'autres termes, il semblerait que la mise en œuvre et l'observation de dispositifs destinés à favoriser l'accrochage des élèves et à encourager leur persévérance soit une piste porteuse pour l'avenir dans la réduction du nombre d'élèves abandonnant l'école sans qualification. Partant du constat que l'institution elle-même génère un processus de décrochage, par exemple en envisageant systématiquement un changement de 
groupe d'apprentissage en cas de difficulté (Lafontaine, Crahay, 2004), la figure du décrocheur interroge dès lors l'ensemble de l'organisation du système éducatif (Boldt, 2004). Les transformations envisagées touchent les problématiques identifiées dans la majorité des cas de décrochage: les cursus comme par exemple, la limitation du redoublement et l'organisation de la scolarité en cycles pluriannuels, les politiques d'orientation et les réseaux prévoyant une prise en charge collective des responsabilités. Cette logique permet de passer de mesures d'urgence sans effet notoire à long terme à l'institution d'une nouvelle normalité (Fischer, 2004). Un certain nombre de ces dispositifs sont notamment basés sur l'élaboration d'alliances éducatives avec des partenaires pour favoriser l'accrochage scolaire notamment auprès des populations fragiles (Hillau, 2013 ; Tièche-Christinat, Gilles, 2013), ces alliances s'avérant indispensables lorsqu'il s'agit d'associer prévention et remédiation pour les jeunes ayant amorcé un processus de décrochage (Lafontaine, Crahay, 2004). Les préconisations des chercheurs vont largement dans le sens d'un ancrage fort des politiques publiques au sein des territoires, avec une mobilisation forte de tous les acteurs locaux dans l'objectif de persévérance scolaire de tous les élèves (Boudesseul, 2013 ; Perron, Veillette, 2011).

\section{Conclusion : vers une approche « orientée activité » du décrochage scolaire}

L'approche du phénomène du décrochage scolaire a évolué dans les recherches récentes, d'un regard sur «l'élève décrocheur » qu'il convenait de repérer afin de lui faire bénéficier de mesures pour le ramener au sein du système scolaire, vers une analyse des politiques publiques d'éducation pointant ce qui au sein de ces politiques pouvait provoquer l'abandon scolaire de certains élèves, dans le but d'en améliorer le fonctionnement.

$\mathrm{Au}$ sein de ce paradigme, et partant du constat que les phénomènes éducatifs sont des processus complexes, multifactoriels, peu prévisibles, impliquant de nombreux tiers et finalement peu déterminés, il convient à notre avis de se pencher sur les démarches qui privilégient l'observation fine des situations elles-mêmes, dans le cadre de ce que les auteurs appellent des approches «orientées activité » (Durand, De Saint-Georges, Meuwly-Bonte, 2006 ; Yvon, Durand, 2012). En l'occurrence, le fait que la genèse du décrochage scolaire passe par une phase de «décrochage de l'intérieur» incite à se focaliser sur ce qui se joue dans les interactions en classe, en prenant en compte l'activité réelle. Si l'on fait le choix de ce type $d$ 'approche «orientée-activité », il s'agit alors, au-delà et en complément des préconisations d'ordre structurel, d'envisager la réalité du processus de décrochage au sein de la classe, au travers de méthodes ancrées dans une épistémologie de l'action, visant à maintenir une proximité avec le réel de l'activité des individus, enseignants et élèves (Flavier, Moussay, Méard, 2013 ; Veyrunes, Gal-Petitfaux, Durand, 2009). C'est le cas par exemple de l'étude de Vors et Gal-Petitfaux (2008) qui examinent l'activité d'une classe en réseau ambition réussite (RAR) en cours d'EPS, et constatent chez les élèves une alternance de moments d'engagement et de décrochage. De la même façon, Guérin, Pasco et Riff (2008) parviennent à mettre en évidence la part publique et la part dissimulée de l'activité d'un élève identifié comme «décrocheur de l'intérieur» lors d'un cours de mathématiques. Ils mettent en lumière des préoccupations simultanées et contradictoires chez ce jeune scolarisé, 
correspondant à des moments de décrochage, alternant avec d'autres activités en rapport avec les enseignements.

Nous considérons, à l'issue de cette revue de littérature, que le décrochage scolaire ne constitue pas une manifestation repérable concernant une population distincte conjuguant un certain nombre de caractéristiques, en rapport avec des conditions familiales et institutionnelles déterminées mais plutôt un processus co-construit «en situation», notamment dans les interactions entre enseignants et élèves au sein de la classe (Bautier et al., 2002 ; Bonnéry, 2003 ; Méard, 2013). De là, l'exploration de cette approche « orientéeactivité » et les considérations épistémologiques et méthodologiques qui en découlent nous paraissent heuristiques dans la mesure où elles permettent une analyse fine de la complexité $\mathrm{du}$ processus de décrochage, comprise comme une co-construction de l'activité des enseignants et des élèves au sein de l'école d'aujourd'hui. D'autre part, en complément des recherches portant sur les améliorations structurelles des systèmes scolaires, il nous semble important de nous pencher sur la manière dont la prévention du décrochage impacte l'activité réelle des professionnels enseignants, et comment ces derniers s'y prennent au quotidien pour favoriser la persévérance de leurs élèves.

Au-delà, le principal bénéfice de cette approche tient selon nous en la restauration du pouvoir d'agir des acteurs qu'elle autorise (Clot, 2008). Rompant avec la logique qui consiste, au travers de directives ministérielles ou académiques, à proposer des dispositifs spécifiques et juxtaposés dans les établissements, en réponse à tel ou tel facteur de risque, elle réintègre le rôle de l'enseignant dans la classe, le propre sens qu'il donne à son activité, sa propre efficience, ses propres prérogatives, associant dès lors dans la même problématique la question de la prévention du décrochage scolaire des élèves à celle de la prévention du décrochage professionnel de l'enseignant.

Néanmoins, cette approche «orientée activité », si elle nous semble nécessaire, ne constituerait pas non plus un aboutissement. Quand bien même serait-elle massivement mise en œuvre, il resterait la difficulté d'analyses longitudinales de processus microscopiques à court et moyen terme (quelques mois) en classe à articuler à un processus qui se manifeste sur le long terme : le décrochage scolaire. On peut donc avancer que, suite aux multiples données fournies notamment par la sociologie et la psychologie, les voies de compréhension de ce phénomène impliqueront à l'avenir des approches méthodologiques multiples, associant par exemple des analyses cliniques et des récits de vie à visée biographique.

Françoise Bruno, Christine Félix, Frédéric Saujat

Apprentissage, didactique, évaluation, formation (ADEF - ÉA 4671)

Aix-Marseille Université, Ifé-ENS Lyon 


\section{Bibliographie}

Alexander, K.-L., Entwisle, D.-R., Kabbani, N.-S. (2001). The dropout process in life course perspective : early risk factors at home and school. Teachers College Record, $\mathrm{n}^{\circ} 103, \mathrm{p} .760$ 822.

Aloise-Young, P.-A., Chavez, E.-L. (2002). Not all school dropouts are the same: Ethnic differences in the relation between reason for leaving school and adolescent substance use. Psychology in the Schools, $\mathrm{n}^{\circ} 39(5), \mathrm{p} .539-547$.

Anderman, E.-M. (2002). School effects on psychological outcomes during adolescence. Journal of Educational Psychology, n94(4), p.795-809.

Anderson, A., Hamilton, R. Hattie, J. (2004). Classroom climate and motivated behaviour in secondary schools. Learning Environments Research, nº 7 , p.211-225.

Aston N.-M., McLanahan S.-S. (1991). Family Structure, Parental Practices, and High School Completion. American Sociological Review, n56, p.309-320.

Aston, N.-M., McLanahan, S.-S. (1994). Family structure, residential mobility, and school dropout: A research note. Demography, n³1 (4), p.575-584.

Baker, J.-A.; Derrer, R.-D.; Davis, S.-M.; Dinklage-Travis, H.-E.; Linder, D.-S., Nicholson, M.-D. (2001). The flip side of the coin: Understanding the school's contribution to dropout and completion. School Psychology Quarterly, n 16(4), p.406-426.

Balas, G. (2012). Lutter contre le décrochage scolaire. Vers une nouvelle action publique régionale. Paris : Fondation Jean Jaurès.

Battin-Pearson, S., Newcomb, M.-D., Abbott, R.-D., Hill, K.-G., Catalano, R.-F., Hawkins, J.D. (2000). Predictors of early high school dropout: a test of five theories. Journal of Educational Psychology, n 92 , p.568-582.

Bautier, E., Rochex, J.-Y. (1997). Apprendre : des malentendus qui font la différence, in La scolarisation de la France, J.P. Terrail (éd.). Paris : La Dispute, p.105-122.

Bautier, E., Terrail, J.-P., Branca, S., Bonnéry, S., Bebi, A., Lesort, B. (2002). Décrochage scolaire: genèse et logique des parcours. Rapport de recherche pour la DPD/ MEN.

Bernard, P.-Y. (2007). La construction du décrochage scolaire comme problème public. In Actes du colloque international La fabrique de populations problématiques par les politiques publiques. MSH Ange-Guépin, Nantes, France.

Bernard, P.-Y. (2011a). Le décrochage scolaire. Paris : Presses universitaires de France.

Bernard, P.-Y. (2011b). Le décrochage des élèves du second degré: diversité des parcours, pluralité des expériences scolaires. Les Sciences de l'éducation-Pour l'Ėre nouvelle, n45(4), p.75-97. 
Blanchard, C., Pelletier, L., Otis, N. Sharp, E. (2004). Rôle de l'autodétermination et des aptitudes scolaires dans la prédiction des absences scolaires et l'intention de décrocher. Revue des sciences de l'éducation, $\mathrm{n}^{\circ} 30(1), \mathrm{p} .105-123$.

Blaya, C. (2009). L'absentéisme des collégiens: prévalence et caractéristiques. Les Sciences de l'éducation-Pour l'Ère nouvelle, $\mathrm{n}^{\circ} 42(4)$, p.39-58.

Blaya, C. (2010). Décrochages scolaires - L'école en difficultés. Bruxelles : De Boeck.

Blaya, C. (2013). Le décrochage scolaire dans les pays de l'OCDE. Regards croisés sur l'économie, $\mathrm{n}^{\circ} 2$, p.69-80.

Blaya, C., Fortin, L. (2011). Les élèves français et québécois à risque de décrochage scolaire: comparaison entre les facteurs de risque personnels, familiaux et scolaires. L'Orientation scolaire et professionnelle, $\mathrm{n}^{\circ} 40(1), \mathrm{p} .55-85$.

Blaya, C., Gilles, J.-L., Plunus, G., Tièche-Christinat, C. (2011). Accrochage scolaire et alliances éducatives: vers une intégration des approches scolaires et communautaires. Éducation et francophonie, $\mathrm{n}^{\circ} 39(2), \mathrm{p} .227-249$.

Blaya, C., Hayden, C. (2003). Construction sociale du refus de l'école: processus de nonscolarisation, de déscolarisation et de décrochage scolaire en France et en Angleterre. Rapport de recherche pour la Direction de la Programmation et du Développement, (2000012).

Boldt, S. (2004). Décrocheurs/raccrocheurs en Irlande. Comment interrogent-ils le système éducatif ? Revue internationale d'éducation de Sèvres, $\mathrm{n}^{\circ} 35, \mathrm{p} .67-80$.

Bonnéry, S. (2001). Décrochage et raccrochage au collège: l'entrée en sixième et l'effet loupe des élèves de classes relais. Actes du colloque Production/réduction des inégalités dans/hors l'École organisé par l'association Défendre et Transformer l'École Pour Tous. https://halshs.archives-ouvertes.fr/halshs-00680151 consulté le 2 novembre 2016.

Bonnéry, S. (2003). Le décrochage scolaire de l'intérieur : interaction de processus sociaux, cognitifs, subjectifs et langagiers. Les sciences de l'éducation. Pour l'ère nouvelle, $\mathrm{n}^{\circ} 36$ (1), p.39-58.

Bonnéry, S. (2004a). Décrochage cognitif et décrochage scolaire, in Glasman, D., Oeuvrard, F. (dir.). La déscolarisation. Paris : La Dispute. p.135-149.

Bonnéry, S. (2004b). Le décrochage scolaire en France : un «problème social » émergent ? Revue internationale d'éducation de Sèvres, n³5, p.81-88.

Boudesseul, G. (2013) Du décrochage à la réussite scolaire: Expériences française et internationales. Paris : L'Harmattan, 
Bourdieu P., Champagne P. (1993), Les exclus de l'intérieur, in P. Bourdieu, La misère du monde. Paris : Seuil. p.597-603.

Broccolichi, S. (1995). Orientations et ségrégations nouvelles dans l'enseignement secondaire. Sociétés contemporaines, ${ }^{\circ} 21$, p.15-27.

Broccolicchi, S., Ben Ayed, C. (1999). L'institution scolaire et la réussite de tous aujourd'hui : pourrait mieux faire. Revue Française de Pédagogie, n¹29, p.39-51.

Brown, T.-M., Rodriguez, L.-F. (2009). School and the Co-Construction of Dropout. International Journal of Qualitative Studies in Education, ${ }^{\circ} 22(2)$, p.221-242.

Bruno, F., Méard, J., Walter, E., (2013). Les dispositifs français de lutte contre le décrochage scolaire en collège : ce qui est prescrit et ce qui est mis en œuvre. L'Orientation Scolaire et Professionnelle, $\mathrm{n}^{\circ} 42$ (4), p.439-459.

Cairns, R.-B., Cairns, B.-D., Neckerman, H.-J. (1989). Early school dropout : Configurations and determinants. Child Development, ${ }^{\circ} 60, \mathrm{p} .1437-1452$.

Chapman, C., Laird, J., Ifill, N., Kewal Ramani, A. (2011). Trends in High School Dropout and Completion rates in the United States : 1972-2009. National center of education statistics (Compendium report).

Christenson, S., Thurlow M. (2004). School dropout : prevention, considerations, interventions, and challenges. Current directions in psychological sciences, $\mathrm{n}^{\circ} 13,1, \mathrm{p} .36-39$

Clot, Y. (2007). De l'analyse des pratiques au développement des métiers. Éducation et didactique, $\mathrm{n}^{\circ} 1(1), \mathrm{p} .83-93$.

Clot, Y. (2008). La recherche fondamentale de terrain, une troisième voie. Éducation permanente, $\mathrm{n}^{\circ} 177$, p.67-78.

Coleman, J.-S., Campbell, E.-Q., Hobson, C.-J., McPartland, J., Mood, A.-M., Weinfeld, F.D.,York, R. (1966). Equality of educational opportunity. Department of Education, Washington, dc, 1066-5684.

Colombo, M. (2010). Dispersione scolastica e politiche per il successo formativo. Dalla ricerca sugli early school leavers alle proposte di innovazione. Trento : Erickson.

Commission Européenne (2011). Proposition de recommandations du conseil concernant les politiques de réduction de l'abandon scolaire. Bruxelles : Commission Européenne.

Cossette, M.-C., Potvin, P., Marcotte, D., Fortin, L., Royer, É., Leclerc, D. (2004). Le risque de décrochage scolaire et la perception du climat de classe chez les élèves du secondaire. Revue de psychoéducation et d'orientation, $\mathrm{n}^{\circ} 33(1), \mathrm{p} .117-136$. 
Coudrin, C. (2006). Devenir des élèves neuf ans après leur entrée en sixième. Note d'information. Paris : Direction de l'Évaluation et de la Prospective.

Darling-Hammond, L. (1999). Teacher quality and student achievement: A review of state policy evidence. Seattle, WA: Center for the Study of Teaching and Policy, University of Washington.

Davis, K.-S., Dupper, D.- R. (2004). Student-teacher relationships: An overlooked factor in school dropout. Journal of human behavior in the social environment, $\mathrm{n}^{\circ}$ 9(1-2), p.179-193.

Dorn, S. (1996). Creating the dropout : an institutional and social history of school failure. Wesport, CN : Praeger.

Douat, E. (2011). L'école buissonnière. Paris : La dispute.

Durand, M., de Saint-Georges, I., Meuwly-Bonte, M. (2006). Le curriculum en formation des adultes: argumentation pour une approche «orientée-activité». Raisons éducatives, $\mathrm{n}^{\circ} 10$, p.185-202.

Englund, M., Egeland, B., Collins, W.-A. (2008). Exceptions to high school dropout predictions in a low-income sample : do adults make a difference? Journal of Social Issues, $\mathrm{n}^{\circ} 64(1), \mathrm{p} .77-94$.

Esterle-Hedibel, M. (2003). Des élèves qui n'en sont plus; les arrêts de scolarité avant 16 ans. Les sciences de l'Éducation pour l'ère nouvelle, n³6(1), p.15-38.

Fallu, J.-S., Janosz, M. (2003). La qualité des relations élève-enseignants à l'adolescence: un facteur de protection de l'échec scolaire. Revue de psychoéducation et d'orientation, $\mathrm{n}^{\circ} 32(1)$, p.7-29.

Fischer, L. (2004). L'abandon scolaire en Italie. Revue internationale d'éducation de Sèvres, $35,31-42$

Flavier, E., Moussay, S., Méard, J. (2013). Quand l'initiative individuelle des professionnels devient la pierre angulaire des dispositifs collectifs locaux de lutte contre le décrochage scolaire. In Actes du Colloque international en Education. Enjeux actuels et futurs de la formation et de la profession enseignante. CRIFPE. Montréal, mai.

Fortin, L., Picard, Y. (1999). Les élèves à risque de décrochage scolaire : facteurs discriminants entre décrocheurs et persévérants. Revue des Sciences de l'Education, n²5(2), p.359-374.

Fortin, L., Royer, E., Marcotte, D., Potvin, P., Yergeau, E. (2004). La prédiction du risque de décrochage scolaire au secondaire : facteurs personnels, familiaux et scolaires. Revue Canadienne des sciences du comportement, $\mathrm{n}^{\circ} 36(3), \mathrm{p} .219-231$. 
Fortin, L., Marcotte, D., Potvin, P., Royer, E., Joly, J. (2006). Typology of Students at Risk of Dropping Out of School: Description by Personal, Family and School Factors. European Journal of Psychology of Education, n²1(4), p.363-383.

Fortin, L., Marcotte, D., Diallo, T., Potvin, P., Royer, E. (2013). A multidimensional model of school dropout from an 8-year longitudinal study in a general high school population. European Journal of Psychology of Education, $\mathrm{n}^{\circ} 28$ (2), p.563-583.

Franklin, C., Streeter, C.-L. (1995). Assessment of middle class youth at-risk to dropout: school, psychologist and family correlates. Children and Youth Services Review, n ${ }^{\circ} 17, \mathrm{p} .433-$ 448.

Geay, B. (2003). Du « cancre » au « sauvageon ». Les conditions institutionnelles de diffusion des politiques «d'insertion » et de " tolérance zéro ». Actes de la recherche en sciences sociales, $\mathrm{n}^{\circ} 4$, p.21-31.

Glasman, D. (2011). Le décrochage scolaire. Paris : Presses universitaires de France.

Glasman, D., Oeuvrard, F. (2011). La déscolarisation. Paris : La Dispute, 2011, $2^{\text {ième }}$ édition ( $1^{\text {ère } e ́ d i t i o n: ~ 2004) . ~}$

Gleason, P., Dynarski, M. (2002). Do We Know Whom to Serve? Issues in Using Risk Factors to Identify Dropouts. Journal of Education for Students Placed at Risk, n7(2), p.2541.

Guérin, J., Pasco, D., Riff. J. (2008). Activités dissimulée et publique d'un élève décrocheur en mathématiques. Les Sciences de l'éducation - Pour l'Ère nouvelle, n41, p.63-82.

Guigue, M. (2013). L'émergence d'expétiences locales, enjeux pour les institutions et pour les acteurs. In Gérard Boudesseul, Du décrochage à la réussite scolaire: Expériences française et internationales. Paris : L'Harmattan, p.39-52.

Hammond, C., Linton, D., Smink, J., Drew, S. (2007). Dropout risk factors and exemplary programs. Retrieved from National Dropout Prevention Center, Communities In Schools, Inc. Website:

http://www.dropoutprevention.org/resource/major_reports/communities_in_schools/Dropout \%20Risk\%20Factors\%20and\%20Exemplary\%20Programs\%20FINAL\%205-16-07.pdf

Hardre, P., Reeve, J. (2003). A motival model of rural students' intentions to persist in, versus drop out of, high school. Journal of Educational Psychology, n 95-2, p.347-356.

Hauser, R.-M., Solon J.-S., Pager, D.-I. (2004). High school dropout, race-ethnicity, and social background from the 1970s to the 1990s. in Gary Orfield (dir.). Dropouts in America : Confronting the Graduation Rate Crisis. Cambridge, Massachusetts: Harvard Educational Publishing Group. p.85-106. 
Hayden, C., Blaya, C. (2008). Lost in transition? A comparison of early drop out from education and training in England and France. The International Journal on School Disaffection, $\mathrm{n}^{\circ}$ 6(1), p.19-24.

Hickman, G.-P., Bartholomew, M., Mathwig, J., Heinrich, R.-S. (2008). Differential developmental pathways of high school dropouts and graduates. The Journal of Educational Sciences, $\mathrm{n}^{\circ} 102(1)$, p.3-14.

Hillau, B. (2013). Le local comme échelon de construction de l'action publique, In Gérard Boudesseul, Du décrochage à la réussite scolaire: Expériences française et internationales. Paris : L'Harmattan, p.91-110.

Janosz, M. (2000). L'abandon scolaire chez les adolescents: perspective nord-américaine. VEI enjeux, $\mathrm{n}^{\circ} 122$, p.105-127.

Janosz, M., Archambault, I., Morizot, J., Pagani, L.-S. (2008). School engagement trajectories and their differential predictive relations to dropout. Journal of Social Issues, n ${ }^{\circ} 64(1)$, p.2140 .

Janosz, M., LeBlanc, M. (1996). Pour une vision intégrative des facteurs reliés à l'abandon scolaire. Revue canadienne de psycho-éducation, $\mathrm{n}^{\circ} 25(1)$, $\mathrm{p} .61-88$.

Jimerson, S., Anderson, G., Whipple, A. (2002). Winning the battle and losing the war: examining the relation between grade retention and dropping out of high school. Psychology in the School, $\mathrm{n}^{\circ} 39$ (4), p.441-457.

Knesting, K., Waldron, N. (2006). Willing to Play the Game: How At-Risk Students Persist in School. Psychology in the Schools, n²3(5), p.599-611.

Lafontaine, D., Crahay, M. (2004). Échec et décrochage scolaires en Communauté française de Belgique. Revue internationale d'éducation de Sèvres, n³5, 55-66.

Lagana, M.-T. (2004). Protective factors for inner-city adolescents at risk of school dropout: Family factors and social support. Children \& Schools, ${ }^{\circ} 26(4)$, p.211-220.

Lan, W., Lanthier, R. (2003). Changes in students' academic performance and perception of school and self before dropping out of school. Journal of Education for Students Placed at Risk, n8, p.309-332.

Langenkamp, A.-G. (2010) Academic Vulnerability and Resilience during the Transition to High School : The Role of Social Relationships and District Context. Sociology of Education, n $83(1)$, p.1-19.

Lee, V.-E., Burkam, D.-T. (2003). Dropping out of high school: The role of school organization and structure. American Educational Research Journal, n ${ }^{\circ}$ (2), p.353-393. 
Lehr, C.-A., Hansen, A., Sinclair, M.-F., Christenson, S.-L. (2003). Moving beyond dropout towards school completion: an integrative review of data-based interventions. School Psychology Review, n³2(3), p.342-364.

Lessard, A., Fortin, L., Joly, J., Royer, É., Blaya, C. (2004). Students at-risk for dropping out of school: Are there gender differences among personal, family and school factors? Journal of At-Risk Issues, $\mathrm{n}^{\circ} 10(2), \mathrm{p} .19-27$.

Lessard, A., Fortin, L., Joly, J., Royer, E., Marcotte, D., Potvin, P. (2007). Cheminement de décrocheurs et de décrocheuses. Revue des Sciences de l'Éducation, n³3 (3), p.647-662.

Lessard, A., Fortin, L., Marcotte, D., Potvin, P., Royer, E. (2009). Why Did They Not Drop Out? Narratives from Resilient Students. Prevention Researcher, ${ }^{\circ} 16(3)$, p.21-24.

Malo, C., Sarmiento, J. (2010). Décrocher ou s'accrocher socialement. Rêves socioprofessionnels des jeunes en difficulté de comportement. Sociétés et Jeunesses en Difficulté, $\mathrm{n}^{\circ}$ 9. URL : http://sejed.revues.org/index6714.html

Marcotte, J., Lachance, M.-H., Lévesque, G. (2012). Pleins Feux sur la Persévérance et le Raccrochage. Canadian Journal of Education/Revue canadienne de l'éducation, $\mathrm{n}^{\circ} 34(4)$, p.135-157.

Marcotte, D., Lévesque, N., Fortin, L. (2006). Variations of cognitive distortions and school performance in depressed and non-depressed high school adolescents: a two-year longitudinal study. Cognitive Therapy and Research, n³0, p.211-225.

Marks, G.-N. , Fleming, N. (1999). Early school leaving in Australia: findings from the 1995 year 9 LSAY cohort. Victoria : the Australian council for educational research.

Méard, J. (2013). L'activité des élèves en risque de décrochage scolaire en EPS : la dynamique des interactions en classe. eJRIEPS, n³0, p.99-104.

MEN (1994). Nouveau contrat pour l'école. Paris : MEN. http://www.formapex.com/telechargementpublic/textesofficiels/1994_1.pdf (consultation : 28 octobre 2014).

Millet, M. (2004). Ruptures familiales et « ruptures scolaires » de collégiens de milieux populaires. Actes du colloque : Le devenir des enfants de familles défavorisées en France. Paris.

Millet, M., Thin, D. (2005). Ruptures scolaires. L'école à l'épreuve de la question sociale. Paris : Presses universitaires de France.

Millet, M., Thin, D. (2007). Scolarités singulières et déterminants sociologiques. Revue française de pédagogie, ${ }^{\circ} 4, \mathrm{p} .41-51$. 
Murray, C. (2009). Parent and Teacher Relationships as Predictors of School Engagement and Functioning Among Low-Income Urban Youth. The journal of early adolescence, $\mathrm{n}^{\circ} 29(3)$, p.376-404.

Natriello, G. (1997). Dropouts, school leavers, and truancy. In J. Saha (dir.). International encyclopaedia of the sociology of education. Oxford: Pergamon. p.577-581.

O'Connell, M., Sheikh, H. (2009). Non-Cognitive Abilities and Early School Dropout : Longitudinal Evidence from NELS. Educational Studies, n³5(4), p.475-479.

Perron, M., Veillette, S. (2011). Territorialité, mobilisation des acteurs et persévérance scolaire : le cas du Conseil régional de prévention de l'abandon scolaire au Saguenay-LacSaint-Jean. Économie et Solidarités, n²41(1-2), p.104-127.

Potvin, P., Fortin, L., Marcotte, D., Royer, E., Deslandes P. (2004). Guide de prévention du décrochage scolaire. CTREQ : Québec.

Ribolzi, L. (1984). La scuola incompiuta. Milano : Vita e pensiero.

Robertson, A., Collerette, P. (2005). L'abandon scolaire au secondaire: prévention et interventions. Revue des sciences de l'éducation, n³1(3), p.687-707.

Rodriguez, L.-F. (2010). Preventing Dropout and Promoting Engagement Among Latino Middle and High School Students: Towards a Framework for Understanding and Action. California Association of Latino Superintendents and Administrators (CALSA). University of California Los Angeles, CA.

Rumberger, R. (1995). Dropping out middle school : a multilevel analysis of students and school. American Educational Research Journal, n³2 (3), p.583-625.

Rumberger, R. (2001). Who drops out of school and why. Understanding Dropouts: Statistics, Strategies, and High-Stakes Testing. Washington, D.C. : National Academy Press.

Rumberger, R. (2004). Why students drop out of school. In Gary Orfied (Ed.). Dropouts in America: Confronting the Graduation Rate Crisis. Cambridge, MA: Harvard Education Press. p.131-155.

Rumberger, R. (2011) Dropping out. Cambridge (MA) : Harvard University Press.

Rumberger, R., Lamb, S. (2003). The early employment and further education experiences of high school dropouts: a comparative study of United States and Australia. Economics of Education Review, n²2, p.353-366.

Rumberger, R., Lim, S.-A. (2008). Why students drop out of school: A review of 25 years of research. California Dropout Research Project Report. Santa Barbara, CA, University of California. 
Rumberger, R., Palardy, G. (2005) Test Scores, Dropout Rates, and Transfer Rates as Alternative Indicators of High School Performance. American Educational Research Journal, $\mathrm{n}^{\circ} 42, \mathrm{p} .3-42$.

Sinclair, B.-B., Fraser, B.-J. (2002). Changing classroom environments in urban middle schools. Learning Environments Research, n5, p.301-328.

Saint-Pierre, V., Denault, A.-S.,Fortin, L. (2012). Le risque de décrochage scolaire et la participation à des activités parascolaires à l'école secondaire: effets médiateurs des symptômes dépressifs et des problèmes de comportement extériorisés. Canadian Journal of Education/Revue canadienne de l'éducation, $\mathrm{n}^{\circ} 35(2), \mathrm{p} .379-400$.

Tièche-Christinat, C. (2015). Favoriser la continuité et prévenir la rupture, le rôle d'un dispositif d'accrochage scolaire. Conférence au Colloque du Lasalé, Lausanne, HEP, juillet.

Tièche-Christinat, C., Gilles, J.-L. (2013). Alliances éducatives et accrochage scolaire. Éducation \& Formation, e 300, p.9-14.

Thin, D. (2002). L'autorité pédagogique en question. Le cas des collèges de quartiers populaires. Revue française de pédagogie, ${ }^{\circ} 139$, p.21-30.

Vallerand, R.-J., Fortier, M.-S., Guay, F. (1997). Self-determination and persistence in a reallife setting : toward a motivational model of high-school dropout. Journal of personality and social psychology, $\mathrm{n}^{\circ} 72$ (5), p.1161-1176.

Vanttaja, M., Jarvinen, T. (2006). The Young Outsiders: The Later Life Courses of "Drop-Out Youths. International Journal of Lifelong Education, $\mathrm{n}^{\circ} 25(2)$, p.173-184.

Veyrunes, P., Gal-Petitfaux, N., Durand, M. (2009). Configurations of activity: from the coupling of individual actions to the emergence of collective activity. A study of mathematics teaching situation in primary school. Research papers in Education, n²4(1), p.95-113.

Vors, O., Gal-Petitfaux, N. (2008). Mettre une classe au travail en Réseau Ambition Réussite : des formes typiques d'interaction enseignant-élèves lors de leçons d'EPS, Travail et formation en éducation [En ligne], 2, Consulté le 11 septembre 2013. URL: http://tfe.revues.org/724

Yvon, F., Durand, M. (eds) (2012). Réconcilier recherche et formation par l'analyse de l'activité. Bruxelles : De Boeck.

Zay, D. (2005). Prévenir l'exclusion scolaire et sociale des jeunes. Une approche francobritannique. Paris : Presses universitaires de France. 